\title{
Adaptive Phase in Martensitic Transformation
}

\author{
A. G. Khachaturyan*, S. M. Shapiro** and S. Semenovskaya*
}

\begin{abstract}
It is shown that an adaptive transient phase can be formed along the martensitic transformation path to overcome thermodynamic and nucleation barriers caused by the transformation strain. A possible role of the adaptive phase in the thermal nucleation of the martensite is discussed. The structure of the adaptive phase is predicted from the strain accommodation condition. An example of the cubic $\rightarrow$ tetragonal transformation is considered. The crystal lattice parameters of the adaptive phase are expressed through those of the parent cubic phase and tetragonal normal martensite. It is shown that the $7 \mathrm{R}$ martensite in $\beta^{\prime} \mathrm{NiAl}$ alloys is an example of the adaptive martensite.
\end{abstract}

(Received October 21, 1991)

Keywords: martensite, habit plane, accommodation strain, nucleation, stacking faults, incommensurate structures

\section{Introduction}

A crystal lattice mismatch between a parent and martensite phase imposes a severe constraint on the transformation path of the martensitic transformation. The transformation can proceed only along a path providing almost complete accommodation of the mismatch-induced strain. This mainly occurs through a formation of martensite plates. According to Ref. (1)-(3), a formation of a coherent martensite plate consisting of periodically alternating lamellae of two twin-related orientation variants of the martensite (Fig. 1) results in a vanishing the volume dependent elastic energy. The volume-dependent part of the strain energy is the only one which could affect the phase equilibrium in the classical thermodynamics. The volume fractions of two orientation variants, $\omega$ and $1-\omega$, respectively, are determined from a condition that they provide the macroscopic shape change described by an invariant plane strain. The remaining elastic energy proves to be proportional to the habit plane surface:

$$
E_{\text {surf }}^{\mathrm{el}} \sim \mu \varepsilon_{0}^{2} S \lambda
$$

where $\lambda=d_{1}+d_{2}$ is an alternation period, $d_{1}$ and $d_{2}$ are thicknesses of lamellae, $\mu$ is a typical shear modulus, $\varepsilon_{0}$ is a typical crystal lattice mismatch and $S$ is the plate/matrix interfacial area. This surface-dependent strain energy, $E_{\text {surf }}^{\text {el }}$, renormalizes the total interfacial energy. On the other hand, the twin-related lamellae introduce an additional energy term caused by surface energy of interlamellar boundaries:

$$
E_{\text {inhom }}=\frac{2 \gamma_{\mathrm{tw}}}{\lambda} V
$$

where $\gamma_{\mathrm{tw}}$ is the twin surface energy. Being volume-dependent it can be included into the total free energy of the

* Department of Mechanics and Materials Science, Rutgers University, P.O. Box 909, Piscataway, N.J. 08855, U.S.A.

** Brookhaven National Laboratory, Upton, N.Y. 11973, U.S.A. martensitic plate. The elastic energy (1) and the surface energy (2) have an opposite effect on the martensite morphology. The smaller the period $\lambda$, the less the strain-induced part of the surface energy (1). On the other hand, the decrease of the period $\lambda$ increases surface energy (2). Competition of these two energies determines the equilibrium twin thickness. Below we consider a case of small twin surface energy and investigate its effect on the martensite morphology.

\section{Twin Structure Miniaturization}

If surface energy coefficient $\gamma_{\mathrm{tw}}$ is very small, the twin thickness is determined by the elastic strain energy (1) only. It decreases as long as the thickness $\lambda$ decreases. Let us consider a minimum elastic energy morphology described by the period $\lambda$ which reaches the smallest possible value. This value should be consistent with the invariant plane condition and a natural crystallographic requirement that the lamellar thicknesses and value $\lambda$ are multiples of interplanar distance, $a_{\mathrm{tw}}$, of the twinning plane. In other words, the following conditions have to be met:

$$
\frac{d_{1}}{d_{2}}=\frac{\omega}{1-\omega}, \quad d_{1}=m a_{\mathrm{tw}}, \quad d_{2}=n a_{\mathrm{tw}}, \quad d_{1}+d_{2}=\lambda
$$

where $m$ and $n$ are small integers. The periodic distribution of microtwins with atomic scale periods (3) is just another geometrical description of a new long-period phase. Its ideal homogeneous periodic atomic structure is related to the atomic structure of the normal martensite phase by an appropriate shuffling of crystal planes (which imitates the twinning) combined with a certain distortion of these planes and their interplanar distance (which imitates the homogeneous parent $\rightarrow$ martensite phase strain for the normal martensite). We call this phase the "adaptive" phase. The concept of the adaptive phase has been introduced in Ref. (4). The period $\lambda$ shown in Fig. 1(a) is the new long-period of the adaptive lattice. It, of course, should be remembered that microtwinning, unlike "macrotwinning"' may slightly affect the 


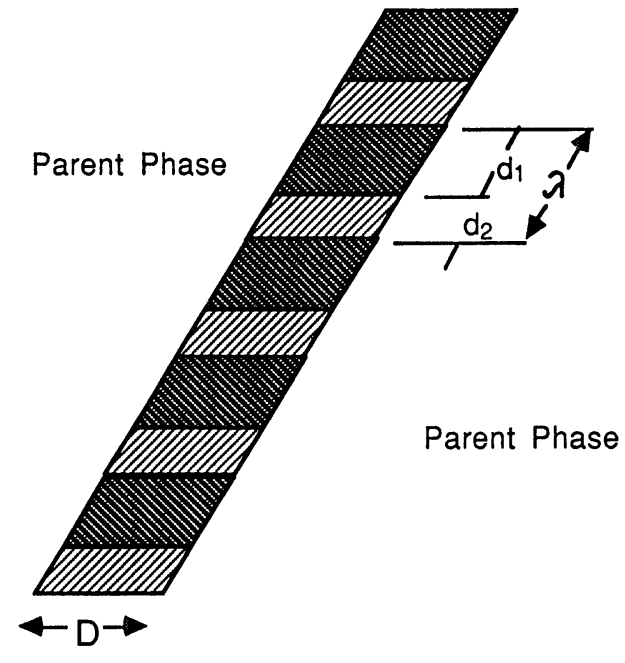

(a)

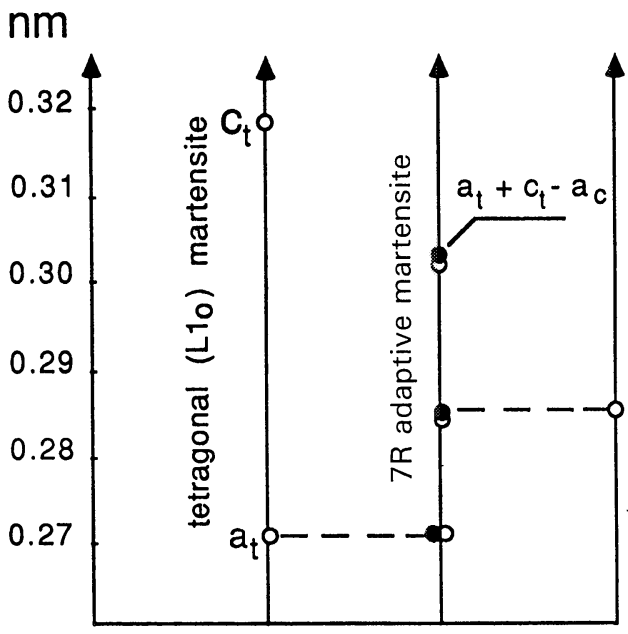

(b)

Fig. 1 (a) A schematic representation of the martensitic phase plate composed by twin-related lamellae of two orientation variants of the martensitic phase. The appropriate $d_{1}$ to $d_{2}$ ratio completely accommodates the martensite/parent phase macroscopic transformation strain mismatch along the habit plane. (b) Relation between observed crystal lattice parameters of $\mathrm{NiAl}, c_{\mathrm{t}}$ and $a_{\mathrm{t}}$, of the normal tetragonal $\mathrm{L1}_{0}$ martensite (left axis, open circles), observed lattice parameters of the pseudo-orthorhombic 7R martensite (medium axis, open circles), the cubic B2 parent phase, $a_{\mathrm{c}}$, (right axis, open circles) and the calculated according to eq. (8) parameters of the adaptive martensite (medium axis, filled circles). Dash line emphasizes equality, $a_{\mathrm{ad}}=a_{\mathrm{c}}$, $c_{\mathrm{ad}}=a_{\mathrm{t}}$. Figure illustrates an excellent agreement with the prediction (8) showing that the $7 \mathrm{R}$ martensite is an adaptive phase.

crystal lattice parameters of the adaptive phase because of the interaction between microtwin boundaries. This interaction may be substantial since a distance between the nearest microtwin boundaries in the adaptive phase is microscopically small.

An adaptive phase can be regarded as any other metastable conventional phase. Its bulk free energy ex- ceeds the stress-free free energy of the normal martensite by the value, $E_{\text {inhom }}$, given by (2). Therefore, the adaptive martensite formation is expected only below the temperature of its metastable equilibrium with the parent phase, $T_{\text {ad }}$. It is, of course, below the temperature of the normal martensite $\rightarrow$ parent phase stress-free equilibrium, $T_{0}$. An adaptive phase concept, actually, allows us to resolve a problem of the homogeneous thermal nucleation of the martensitic phase. Below $T_{\text {ad }}$, the system may just bypass the insurmountable strain-induced nucleation barrier for the normal martensite phase by nucleating the metastable adaptive phase instead of normal martensite. The nuclei of the adaptive phase may later easily transform to the normal martensite during the growth stage when the microtwin thickness reaches macroscopic size since the coarsening kinetics, unlike nucleation, does not require overcoming the volume-dependent elastic barriers. If the adaptive phase nucleation mechanism is assumed, then the $M_{\mathrm{s}}$ temperature of the conventional martensitic transformation is equal to $T_{\text {ad }}$, the temperature of the equilibrium between the adaptive and parent phases.

The smaller the twin surface energy, $\gamma_{\mathrm{tw}}$, the more stable is the adaptive martensite since a positive contribution to the adaptive phase free energy (2) is smaller. Depending upon the twin surface energy, different cases can be expected: (i) If $\gamma_{\mathrm{tw}}$ is very low, the adaptive martensite may be sufficiently stable within a wide area of the phase diagram, (ii) if $\gamma_{\mathrm{tw}}$ assumes intermediate values, the adaptive phase exists only within a narrow temperature range below $T_{\mathrm{ad}}$, (iii) if $\gamma_{\mathrm{tw}}$ is high, the adaptive phase is formed only as an "activated state" and immediately transforms into the normal martensite. Observation of the adaptive martensite is always expected in the cases (i) and (ii) if it is not hindered by the low driving force, defects or a disorder related to a deviation from the stoichiometry. As will be shown in this paper the case (i) is, probably, related to a formation of the $7 \mathrm{R}$ martensite in the $\beta \mathrm{NiAl}$ alloys.

The crystal lattice of the adaptive martensite has a lower symmetry than that of the normal (nontwinned) martensite. A long-period unit cell of the adaptive phase is always related to the transformation-related unit cell of the parent phase by an invariant plane strain. Fulfillment of this condition is a fingerprint of the adaptive phase since it is hard to imagine this condition to be fulfilled accidentally.

The second feature of an adaptive phase is that its shuffling (microtwinning) planes must always be parallel to the twinning (mirror) plane relating two orientation variants of the normal martensite. For example, for a cubic $\rightarrow$ tetragonal transformation it is the (110) parent plane.

The third feature is less obvious. According to (3), the $\omega /(1-\omega)$ ratio is a simple fraction, $m / n$. On the other hand, this ratio is determined by the invariant plane requirement and, thus, is expressed independently through the crystal lattice parameters of the parent phase and normal martensite. These two ratios can be brought close but not equal to each other. The only way to eliminate 
the mismatch between two ratios is to insert faults in the regular microtwin thickness alternation. The aperiodic faulting should result in shifts of the adaptive phase diffraction spots from the positions determined by the rational period, $\lambda=a_{\mathrm{tw}}(m+n)$. The effect is similar to the effect of stacking faults ${ }^{(5)}$ or correlated faults in a layer structure $^{(6)}$. A diffraction from the faulted structure of the adaptive martensite is distinguished from that of an incommensurate phase by a dependence of superlattice spots positions of the adaptive martensite on the Brillouin zone. Such a dependence should not be observed in true incommensurate or commensurate structures.

Finally, a typical feature of the adaptive martensite is a sensitivity of its crystal structure to the applied stress. The stress affects differently the two orientational variants depending on a mutual orientation of their tetragonality axis with respect to the principle axes of the stress tensor. The stress suppresses one variant and promotes the other. As a result, the ratio $\omega /(1-\omega)$, and, thus, the period $\lambda$, change. Large stress can even completely eliminate one of the variants and, therefore, transform the adaptive martensite to the normal martensite.

\section{Adaptive Phase in the Cubic $\rightarrow$ Tetragonal Martensitic Transformation}

We now consider an important particular case of the adaptive phase in the cubic $\rightarrow$ tetragonal martensitic transformation. The transformation generates three orientation variants whose tetragonal axes are parallel to one of the three directions, $[100]_{c},[010]_{c}$ and $[001]_{c}$. The crystal lattice rearrangements producing the orientation variants are described by three stress-free strain tensors, $\varepsilon(1)_{i j}^{0}, \varepsilon(2)_{i j}^{0}, \varepsilon(3)_{i j}^{0}$. Since a martensite crystal is a sandwich composed of alternating twin-related lamellae of two variants of the tetragonal phase, then the total stressfree macroscopic shape change is described by the average of the respective strains:

$$
\bar{\varepsilon}_{i j}=\omega \varepsilon(1)_{i j}^{0}+(1-\omega) \varepsilon(2)_{i j}^{0}
$$

where

$$
\begin{gathered}
\varepsilon(1)_{i j}^{0}=\left(\begin{array}{ccc}
\varepsilon_{33}^{0} & 0 & 0 \\
0 & \varepsilon_{11}^{0} & 0 \\
0 & 0 & \varepsilon_{11}^{0}
\end{array}\right), \quad \varepsilon(2)_{i j}^{0}=\left(\begin{array}{ccc}
\varepsilon_{11}^{0} & 0 & 0 \\
0 & \varepsilon_{33}^{0} & 0 \\
0 & 0 & \varepsilon_{11}^{0}
\end{array}\right), \\
\varepsilon_{33}^{0}=\frac{c_{\mathrm{t}}-a_{\mathrm{c}}}{a_{\mathrm{c}}}, \quad \varepsilon_{11}^{0}=\frac{a_{\mathrm{t}}-a_{\mathrm{c}}}{a_{\mathrm{c}}}
\end{gathered}
$$

$a_{\mathrm{c}}$ is the crystal lattice parameter of the parent phase, $a_{\mathrm{t}}$ and $c_{\mathrm{t}}$ are parameters of the normal martensite in the stress-free state. The mismatch tensor, $\bar{\varepsilon}_{i j}$, is an invariant plane strain if one of its components vanishes and the remaining two components have an opposite sign. This occurs when

$$
\omega=\varepsilon_{11}^{0} /\left(\varepsilon_{11}^{0}-\varepsilon_{33}^{0}\right)>0
$$

and $\varepsilon_{33}^{0}$ and $\varepsilon_{11}^{0}$ have different signs. Then

$$
\bar{\varepsilon}_{11}=0, \quad \bar{\varepsilon}_{22}=\varepsilon_{33}^{0}+\varepsilon_{11}^{0}, \quad \bar{\varepsilon}_{33}=\varepsilon_{11}^{0} .
$$

Although the results (6) and (7) are derived in the framework of the linear theory of the martensitic transformation $^{(1)(2)}$, vanishing of one of the diagonal components of the average stress-free strain matrix, $\bar{\varepsilon}_{11}$, and equality of the other component, $\bar{\varepsilon}_{33}$, to $\varepsilon_{11}^{0}$ imposed by an invariant plane constraint are fulfilled for large $\varepsilon_{11}^{0}$ and $\varepsilon_{33}^{0}$ as well. It is clear that the martensitic transformation described by the strain (7) results in the cubic $\rightarrow$ pseudo-orthorhombic crystal lattice rearrangement. Indeed, applying (7) to three cubic axes, $a_{1}=$ $\left(a_{\mathrm{c}}, 0,0\right), \quad a_{2}=\left(0, a_{\mathrm{c}}, 0\right), \quad a_{3}=\left(0,0, a_{\mathrm{c}}\right)$, expresses the crystal lattice parameters of the adaptive phase through that of the normal phases, $a_{\mathrm{c}}, c_{\mathrm{t}}, a_{\mathrm{t}}$ :

$$
a_{\mathrm{ad}}=a_{\mathrm{c}}, \quad b_{\mathrm{ad}}=a_{\mathrm{t}}+c_{\mathrm{t}}-a_{\mathrm{c}}, \quad c_{\mathrm{ad}}=a_{\mathrm{t}}
$$

where the definitions $a_{\mathrm{t}}=a_{\mathrm{c}}\left(1+\varepsilon_{11}^{0}\right), c_{\mathrm{t}}=a_{\mathrm{c}}\left(1+\varepsilon_{33}^{0}\right)$ and $b_{\mathrm{ad}}=a_{\mathrm{c}}\left(1+\varepsilon_{11}^{0}+\varepsilon_{33}^{0}\right)=a_{\mathrm{c}}\left(1+\varepsilon_{11}^{0}\right)+a_{\mathrm{c}}\left(1+\varepsilon_{33}^{0}\right)-a_{\mathrm{c}}=a_{\mathrm{t}}+$ $c_{\mathrm{t}}-a_{\mathrm{c}}$ are utilized. As has been stated above, the relations (8) between crystal lattice parameters of the orthorhombic martensite, normal martensite and the parent cubic phase are fingerprints of the adaptive martensite. In deriving eq. (8) we used an assumption that the crystal lattice rearrangement of the adaptive martensite can be reasonably well described by the alternating macroscopic strains, $\varepsilon(1)_{i j}^{0}$ and $\varepsilon(2)_{i j}^{0}$. This assumption does not affect the equation for $a_{\mathrm{ad}}$ and $c_{\mathrm{ad}}$ but it may affect equation for $b_{\text {ad }}$. The adaptive phase has been discussed in detail in Ref. (4) for $\mathrm{NiAl}$ and $\mathrm{Fe}-\mathrm{Pd}$ alloys. We shall only describe the adaptive phase in $\mathrm{Ni}-\mathrm{Al}$ alloys in this paper.

The cubic $\rightarrow$ tetragonal transformation in $\beta^{\prime} \mathrm{NiAl}$ is characterized by the Bain distortion transforming $\mathrm{CsCl}$ type cubic structure of $\beta^{\prime} \mathrm{NiAl}$ phase to the $\mathrm{L}_{0}$ (CuAuItype) tetragonal structure which we shall regard as normal martensite. The normal martensite has been observed in this system by Enami et al. ${ }^{(7)}$ who have shown that $\beta^{\prime} \mathrm{NiAl}$ alloys containing 62 to 64 at $\% \mathrm{Ni}$ have really $\mathrm{L1}_{0}$ structure. There are all indications that the stacking fault energy of the alloys is very low since the observed $\mathrm{L} 1_{0}$ martensite contains a large quantity of $(111)_{\mathrm{Ll}_{0}}$ stacking faults and thin twins ${ }^{(8)(9)}$. The $(111)_{\mathrm{L}_{0}}$ plane is, actually, the $(110)_{c}$ plane of the B2 structure. An observation of many $(111)_{\mathrm{L}_{0}}$ twins and stacking faults is a manifestation of the low surface energy $\gamma_{\mathrm{tw}}$. Thus, the adaptive martensite in these alloys can be expected. According to Shapiro et $a l .^{(10)}$, for the parent $\mathrm{B} 2 \mathrm{Al}-62.5$ at $\% \mathrm{Ni}$ alloy $a_{\mathrm{c}}=$ $0.2857 \mathrm{~nm}$. Martynov et al. ${ }^{(11)(12)}$ reported the crystal lattice parameters of the tetragonal $\mathrm{L}_{0}$ martensite with $\quad 63.1$ at $\% \mathrm{Ni}: \quad a_{\mathrm{t}}=(1 / \sqrt{2}) a_{\mathrm{Li}_{0}}=0.383 / \sqrt{2}=0.2708$ $\mathrm{nm}, c_{\mathrm{t}}=c_{\mathrm{L} 1_{0}}=0.318 \mathrm{~nm}$. Using these data in (8) gives

$$
\begin{aligned}
& a_{\mathrm{ad}}=a_{\mathrm{c}}=0.2857 \mathrm{~nm} \\
& b_{\mathrm{ad}}=a_{\mathrm{t}}+c_{\mathrm{t}}-a_{\mathrm{c}}=0.2708+0.318-0.2857=0.3031 \mathrm{~nm} \\
& c_{\mathrm{ad}}=a_{\mathrm{t}}=0.2708 \mathrm{~nm} .
\end{aligned}
$$

As shown in Fig. 1(b), these values are in an excellent agreement with the measured values, $a_{7 \mathrm{R}}=0.2845 \mathrm{~nm}$, $b_{7 \mathrm{R}}=0.3022 \mathrm{~nm}, c_{7 \mathrm{R}}=0.2709 \mathrm{~nm}$ for the pseudo-ortho- 
rhombic unit cell of the $7 \mathrm{R}$ martensite ${ }^{(11)}$. The agreement is especially conclusive since the calculation results are very sensitive to the input crystal lattice parameters of the $\mathrm{B} 2$ and $\mathrm{L} 10$ phases. It should be also mentioned that actually the adaptive martensite should be slightly monoclinic because of slight mutual transformationinduced rigid body rotation of the microtwins. Using the same observed values of $a_{\mathrm{c}}, a_{\mathrm{t}}, c_{\mathrm{t}}$ in (5) gives $\varepsilon_{33}^{0}=0.113$ and $\varepsilon_{11}^{0}=-0.052$. Substituting these strain components to (6) gives $\omega=0.31$. If, however, the same strain components are calculated from the observed parameters for the $7 \mathrm{R}^{(10)}$ using eq. (8), then eq. (6) gives $\omega=0.30$. The closest simple fraction $\omega_{0}=m /(m+n)=2 / 7 \approx 0.29$. The corresponding $(5, \overline{2})$ adaptive phase comprises alternating 5- and 2-layer (110) $\mathrm{c} / /(111)_{\mathrm{LI}_{0}}$ microtwins. Such a $(5, \overline{2})$ shuffling exactly reproduces the structure of the $7 \mathrm{R}$ phase. The mismatch between $\omega_{0}=2 / 7$ and $\omega \approx 0.30$, can be accommodated by faulting the periodic $7 R$ sequence. The random faulting would result in shift of the superlattice spots of the 7R structure from their ideal positions corresponding to the rational period, $\lambda=7 a_{\mathrm{tw}}$, the shift being different in different Brillouin zone. This effect was observed in Ref. (10) where it was recognized that such an irregular shift rules out the interpretation of the $7 \mathrm{R}$ structure as an incommensurate periodic structure.

A response of the $7 \mathrm{R}$ martensite to the applied stress is also consistent with an interpretation that it is an adaptive phase. Martynov et al. ${ }^{(11)(12)}$ reported appearance of the $\mathrm{L} 1_{0}$ martensite instead of the $7 \mathrm{R}$ martensite under applied stress, an effect predicted above. It should be mentioned that an assumption that the crystal structure of long-period martensites in nonferrous $\beta$ phase alloys might be determined by a strain accommodation has been made in earlier works summarized and discussed in the review paper by Warlimont and Delaey ${ }^{(13)}$.

\section{Conclusions}

If the adaptive phase, which can be interpreted as miniaturized twinned martensite with twin thicknesses of the order of one or several interplanar distances, is less stable than a homogeneous (untwinned) phase but more stable than the parent phase, it can be regarded as a metastable transient phase. In principle, its thermal nucleation is possible since it does not produce a strain energy increase blocking the thermal nucleation of the normal martensite. The adaptive phase as a transient state may be involved in any martensitic transformation. It can be observed in systems with very low surface energy $\gamma_{\text {tw }}$ of twin boundaries. It is suggested that the $7 R$ martensite in $\beta^{\prime} \mathrm{NiAl}$ is actually an adaptive phase formed in the $\mathrm{B} 2 \rightarrow \mathrm{L} 1_{0}$ transformation under conditions of abnormally low $\gamma_{\mathrm{tw}}$. This conclusion is confirmed by an excellent agreement between the calculated and measured parameters of the $7 R$ phase (the calculation does not include any fitting parameters). As is shown in Ref. (4), the intermediate orthorhombic phase in Fe-Pd alloy observed between the parent fcc phase and normal tetragonal martensite ${ }^{(14)}$ seems to be also an another example of an adaptive phase since its calculated and observed parameters are also in an excellent agreement.

\section{Acknowledgements}

A. G. K. and S. S. are gratefully acknowledge the support from Division of Materials Science, U.S. Department of Energy under grant No. DE-FG05-90ER45430. They also thanks Brookhaven National Laboratory where they spent the summer of 1990 where the most of this work was done. The work in Brookhaven is supported by Division of Materials Science of the U.S. Department of Energy under contract No. DE-AC0276CH00016. The authors are grateful to Professors K. Otsuka and A. Roitburd for very useful discussions.

\section{REFERENCES}

(1) A. G. Khachaturyan: Sov. Phys. JETP, 29 (1969), 557.

(2) A. L. Roitburd: Sov. Phys. Solid State, 10 (1969), 2870.

(3) A. G. Khachaturyan: Theory of Structural Transformations in Solids, Wiley and Sons, New York, (1983).

(4) A. Khachaturyan, S. M. Shapiro and S. Semenovskaya: Phys. Rev., B43 (1991), 10832.

(5) S. Hendricks and E. Teller: Chem. Phys., 10 (1969), 147.

(6) M. S. Paterson: J. Appl. Phys., 23 (1952), 865.

(7) K. Enami, S. Nenno and K. Shimizu: Trans. Japan Inst. Metals, 14 (1973), 161.

(8) S. Rosen and J. A. Goebel: Trans. Met. Soc. AIME, 242 (1968), 722.

(9) V. V. Litvinov, A. A. Arhhangel'skaya and V. V. Poleva: Fiz. Metal Metalloved., 38 (1974), 383.

(10) S. M. Shapiro, B. X. Yang, G. Shirane, Y. Noda and L. E. Tanner: Phys. Rev. Letters, 62 (1989), 1298; Y. Noda, S. M. Shapiro, G. Shirane, Y. Yamada and L. Tanner: Phys. Rev., B42 (1990), 10397.

(11) V. V. Martynov, K. Enami, L. G. Khandros, S. Nenno and A. V. Tkachenko: Phys. Met. Metall., 55 (1983), 136.

(12) V. V. Martynov, K. Enami, L. G. Khandros, A. V. Tkachenko and S. Nenno: Scripta Met., 17 (1983), 1167.

(13) H. Warlimont and L. Delaey: Martensitic Transformations in $\mathrm{Cu}-\mathrm{Ag}$ - and $\mathrm{Au}$-Based Alloys, Progress in Materials Science, 18 (1974), ed. by B. Chalmers, J. W. Christian, T. B. Massalski, Pergamon Press, Oxford.

(14) H. Seto, Y. Noda and Y. Yamada: J. Phys. Soc. Jpn., 59 (1990), 965. 